\title{
Efecto de la salinidad sobre la frecuencia ventilatoria branquial de Dormitator latifrons (Richardson, 1984)
}

\section{Effect of salinity on the branchial ventilatory frequency of Dormitator latifrons (Richardson, 1984)}

\author{
Ana Antonia Zapata ${ }^{1}\left[0\right.$, Fernando Vega-Villasante ${ }^{1}\left[0\right.$, Olimpia Chong-Carrillo ${ }^{1}[0$, Manuel Alejandro \\ Vargas-Ceballos 1 (ㅇ, Daniel Badillo-Zapata ${ }^{1,2 *}$ \\ ${ }^{1}$ Laboratorio de Calidad de Agua y Acuicultura Experimental, Departamento de Ciencias Biológicas, Centro Universitario de la Costa, \\ Universidad de Guadalajara, Av. Universidad de Guadalajara no. 203, Del. Ixtapa, CP. 48280. Puerto Vallarta, Jalisco, México. \\ ${ }^{2}$ Cátedras CONACyT, Consejo Nacional de Ciencia y Tecnología (CONACyT), Ciudad de México, México. \\ *Autor de correspondencia: danielbad00@hotmail.com
}

Nota científica recibida: 13 de marzo de 2019 aceptada: 20 de junio de 2019

RESUMEN. Dormitator latifrons habita en un amplio intervalo de salinidades producto de su ciclo migratorio y reproductivo. El objetivo del trabajo fue evaluar el tiempo de respuesta fisiológica y la sobrevivencia al cambio salino en condiciones de laboratorio. Se registró la frecuencia ventilatoria branquial (FVB) basal de organismos a 33 ups (AS) y en agua dulce 0.3 ups $(A D)$. Se contabilizó la frecuencia ventilatoria branquial de organismos que fueron transferidos AD-AS e inversamente AS-AD $(15,25$, y 33 ups), por triplicado, durante 1 minuto, $1,4,24$ y $48 \mathrm{~h}$. Se encontraron diferencias estadísticas $(p<0.05)$ en los peces transferidos de AD-AS y AS-AD al primer minuto con respecto a FVB basal. En todos los tratamientos los organismos recuperaron la FVB una hora después de ser expuestos a la modificación salina. Se tuvo un $100 \%$ de sobrevivencia en todos los tratamientos, $D$. latifrons tiene rápida respuesta fisiológica a cambios bruscos de salinidad.

Palabras clave: Adaptación, condiciones ambientales, estrés, Pez nativo.

ABSTRACT. Dormitator latifrons inhabits a wide range of salinities product of its migratory and reproductive cycle. Our objective was to evaluate physiological response time and survival to saline change in laboratory conditions. The basal branchial ventilatory frequency (FVB) of organisms at 33 ups (AS) and in fresh water 0.3 ups (AD) was registered. The branchial ventilatory frequency of organisms transferred $A D-A S$ and inversely $A S-A D(15,25$, and 33 ups) was determined, in triplicate, during 1 minute, 1, 4, 24 and $48 \mathrm{~h}$. Statistical differences were found $(p<0.05)$ in the fish transferred from AD-AS and AS-AD at the first minute with respect to basal FVB. In all treatments, organisms returned to baseline one hour after being exposed to saline variation. A survival of $100 \%$ was recorded in all treatments. $D$. latifrons shows a rapid physiological response to sudden changes of salinity.

Key words: Adaptation, environmental conditions, stress, native fish.

\section{INTRODUCCIÓN}

La familia Eleotridae, se componen de alrededor de 150 especies de distribución mundial, que habitan en aguas marinas, salobres y dulces, Dormitator latifrons es representante de esta familia, conocido como dormilón gordo del Pacífico, chopopo, chame, puyeque o popoyote (Nordlie y Haney 1993). Es un pez originario de climas templados y subtropicales, tiene valor comercial en las costas de Ecuador, además que se le puede encontrar en ríos, manglares y en lugares tan extremos como charcas o fuera del agua mientras haya humedad (Vicuña 2010, LópezHuerta et al. 2018). Una característica de esta especie es su alta resistencia fisiológica que le permite sobrevivir en lugares donde se presentan concentraciones bajas de oxígeno disuelto, amplio intervalo de temperatura (Castro-Rivera et al. 2005), variaciones de salinidad (Chang 1984), encontrándose de forma frecuente en aguas salobres (Yáñez-Arancibia 


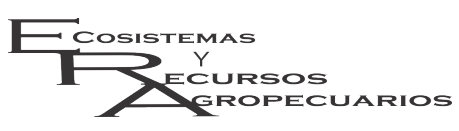

Frecuencia ventilatoria branquial de Dormitator latifrons

Ecosist. Recur. Agropec. 6(18):601-607,2019

y Díaz-González 1977).

En su medio natural $D$. latifrons puede experimentar grandes variaciones de salinidad, pero se tiene poca información sobre la capacidad para enfrentar el estrés osmótico (Todd 1975). La presión osmótica interna de los peces varía dependiendo en el ambiente acuático en el que se desarrollen; los peces de agua dulce poseen una concentración osmótica superior a la de su medio, mientras que en los peces marinos las sales, en los fluidos corporales, se encuentran más diluidas que el agua de mar (Christensen et al. 2017). La osmorregulación es la forma en la que los organismos pueden controlar o mantener estable su medio interno, cuando se exponen a diversas salinidades ambientales, algunos peces solo toleran salinidades similares en las que residen (Kültz 2015). A las especies que tiene la capacidad de sobrevivir dentro de ciertos intervalos de salinidades se les denomina eurihalinas y estas pueden ser de dos tipos, las que toleran y se adaptan a fluctuaciones rápidas de la salinidad externa y las que realizan migraciones en algún momento de su ciclo vital entre medios dulceacuícolas y marinos (Nordlie y Haney 1998). Gran parte del trabajo sobre las respuestas osmorreguladoras se ha realizado principalmente en salmónidos (Serrano et al. 2011). Basado en lo anterior, en este trabajo se evaluó la tolerancia fisiológica al cambio de salinidad, bajo condiciones de laboratorio, de $D$. latifrons, con el objetivo de aportar conocimiento sobre la capacidad de osmorregulación bajo condiciones controladas, y establecer las condiciones ambientales en las que puede ser mantenida en cultivo.

\section{MATERIALES Y MÉTODOS}

Para el desarrollo del estudio se colectaron 200 organismos juveniles de D. latifrons (Laguna El Quelele, Nayarit, México), con peso $10.0 \pm 2.0 \mathrm{~g}$ y talla $13.1 \pm 2.2 \mathrm{~cm}$, los cuales se transportaron en un contenedor de $1000 \mathrm{~L}$ con aireación constante al Laboratorio de Calidad de Agua y Acuicultura Experimental (LACUIC) del Centro Universitario de la Costa de la Universidad de Guadalajara, en Puerto Vallarta, Jalisco, México. Los peces se transfirieron a un es- taque de $1700 \mathrm{~L}$ para cuarentena y mantenimiento a salinidad de 22 ups (unidades prácticas de salinidad), misma salinidad a la que se encontraban en el medio natural. Durante este periodo se suministró un alimento balanceado comercial con $35 \%$ proteínas y $8 \%$ lípidos, aireación constante y temperatura de $24.0 \pm$ $1.0^{\circ} \mathrm{C}$.

Una vez transcurrido el periodo de cuarentena y adaptación al alimento balanceado, se seleccionaron 100 organismos al azar y se colocaron en dos unidades experimentales (UE) de 450 L (50 organismos por UE) con peso y longitud promedio de $8.5 \pm 1.0 \mathrm{~g}$ y $9.5 \pm 1.0 \mathrm{~cm}$ respectivamente. Previo a la colocación de los organismos se realizó un ajuste a la salinidad para mantener salinidades de 0.3 y 33 ups. La modificación de la concentración salina del agua se realizó de manera paulatina incrementando y/o disminuyendo 0.5 ups por hora hasta alcanzar la salinidad deseada partiendo de la salinidad de aclimatación. Durante el periodo del cambio salino se registró la sobrevivencia.

Durante 30 días los peces se mantuvieron a las salinidades ajustadas y se alimentaron con el alimento mencionado previamente, aireación constante y recambio de agua del $20 \%$ semanal. De los tratamientos de 0.3 y 33 ups se seleccionaron al azar seis organismos con peso y longitud promedio de $10.3 \pm 0.5 \mathrm{~g}$ y $11.5 \pm 1.0 \mathrm{~cm}$ respectivamente, los cuales se colocaron de forma individual en acuarios de $20 \mathrm{~L}$, para luego medir la frecuencia ventilatoria branquial (FVB) por medio de los movimientos operculares por minuto, para obtener la frecuencia ventilatoria branquial basal por minuto (FVB basal).

Para determinar la capacidad de los peces de soportar cambios bruscos de salinidad, se seleccionaron al azar tres organismos que se encontraban en el tratamiento de 0.3 ups y se colocaron de forma individual en acuarios de $40 \mathrm{~L}$, aforados a $20 \mathrm{~L}$ de agua a 15 ups, la cual se obtuvo de la mezcla de agua de mar (33 ups) con agua dulce. Los peces se introdujeron de forma directa a la salinidad ajustada, y se mantuvieron por 5 minutos (tiempo de estabilización) para posteriormente proceder a medir la FVB por 60 segundos. Las medidas se realizaron a las $1,4,24$ y $48 \mathrm{~h}$ con un contador manual. La 


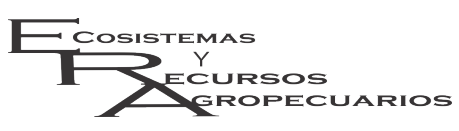

misma metodología se llevó a cabo para determinar la FVB de los peces transferidos de 0.3 a 25 y de 0.3 a 33 para los peces acondicionados a 33 ups y transferidos a 25,15 y 0.3 ups.

\section{Análisis estadístico}

Para determinar las diferencias entre los tratamientos, se realizó una prueba de homogeneidad para después realizar un análisis de varianza. El nivel de significancia se estableció con una $p<$ 0.05. Cuando se detectaron diferencias estadísticas, se utilizó una prueba post hoc de Dunnett para identificar diferencias estadísticas entre tratamientos. Los análisis se realizaron con el programa estadístico GraphPad Prism ${ }^{\circledR}$ 6.01.

\section{RESULTADOS Y DISCUSIÓN}

En el medio natural los organismos acuáticos tienen la capacidad de trasladarse de un lugar a otro hasta encontrar las condiciones fisiológicas adecuadas, cuando las variables fisicoquímicas del agua cambian de manera negativa para su óptimo desarrollo. Pero en condiciones de cultivo los organismos están confinados en espacios restringidos y no pueden desplazarse hacia mejores condiciones ambientales y se genera un estrés que puede desencadenar la aparición de enfermedades o la muerte. El estrés es una condición en la cual el equilibrio dinámico $u$ homeostasis es afectado, amenazado o perturbado por estímulos internos o externos (Corredor y Landines 2009). Los cuales pueden producir respuestas en las que interviene una serie de reacciones bioquímicas y fisiológicas que le permiten recuperar su homeostasis y adaptarse a una nueva situación. Lo que resulta en mayor estado de alerta y movilización de energía (Barreto y Volpato 2004, Blancas-Arroyo et al. 2014).

La FVB en los en peces se utiliza como indicador de estrés o alerta al reconocimiento visual de un depredador (Barreto et al. 2003), como indicador de sobrevivencia de peces o de estrés en ambientes con altos niveles de amonio (Blancas-Arroyo et al. 2014). También se ha reportado que la FVB es una respuesta muy sensible a la perturbación y una
Frecuencia ventilatoria branquial de Dormitator latifrons

Ecosist. Recur. Agropec. 6(18):601-607,2019

técnica no invasiva que no requiere equipos sofisticados para evaluar el bienestar o estados de estrés en los peces (Barreto y Volpato 2004). Al respecto se observó que en condiciones de agua dulce con 0.3 ups, la FVB en juveniles de $D$. latifrons tuvo un promedio de $50.7 \pm 4.2$ ventilaciones por minuto, pero al modificar la salinidad, se tuvieron diferencias estadísticas significativas entre tratamientos ( $p$ $<0.05$ ) (Figura 1). Los tratamientos de $0.3-15$ y 0.333 ups tuvieron un promedio de $75.3 \pm 5.7$ y $103.3 \pm$ 20.2 ventilaciones por minuto, respectivamente; durante el primer minuto de haber sido expuestos al choque osmótico con diferencias estadísticas significativas en la FVB basal (Figura 1a). Después de una hora de la transferencia los peces, solo se observaron diferencias estadísticas entre el tratamiento 0.3-33 ups (Figura 1b). Para las horas subsecuentes la FVB de ningún tratamiento presentó diferencias estadísticas (Figura 1c, d y e).

Sobre la capacidad fisiológica de $D$. latifrons para enfrentar los choques osmóticos, se reporta que el efecto de la salinidad de organismos capturados en agua dulce y transferidos a agua salada a concentraciones de 35, 40, 42, 45 y 50 ups, tuvieron una salinidad media letal de 42 ups (Chang 1984). Con respecto a la respuesta osmoadaptativa de $D$. latifrons, se reporta una supervivencia elevada en la transferencia directa de diferentes concentraciones de agua dulce al agua de mar (Becerra 1997). Lo que indica que este pez es capaz de hipoosmorregular, lo que sugiere su carácter anfídromo, por lo que es capaz de emigrar desde y hacia medios marinos durante todo su ciclo de vida.

En relación con la FVB de los peces transferidos de agua salada (33 ups) ha agua dulce (0.3 ups), se observaron diferencias significativas. Los tratamientos 15-0.3, 25-0.3 y 33-0.3 ups tuvieron una FVB promedio de $79.9 \pm 9.0,94.3 \pm 9.3$ y $84.7 \pm 7.4$ ventilaciones por minuto, respectivamente; al primer minuto de haber sido expuestos al agua dulce, con diferencias significativas (Figura 2a). A los 60 min se encontraron diferencias estadísticas significativas ( $p$ $<0.05$ ) con los tratamientos $15-0.3$ y $33-0.3$ ups con una FVB de $64.3 \pm 4.0$ y $79.7 \pm 1.5$ ventilaciones por minuto, respectivamente (Figura $2 \mathrm{~b}$ ). Por otra parte, 

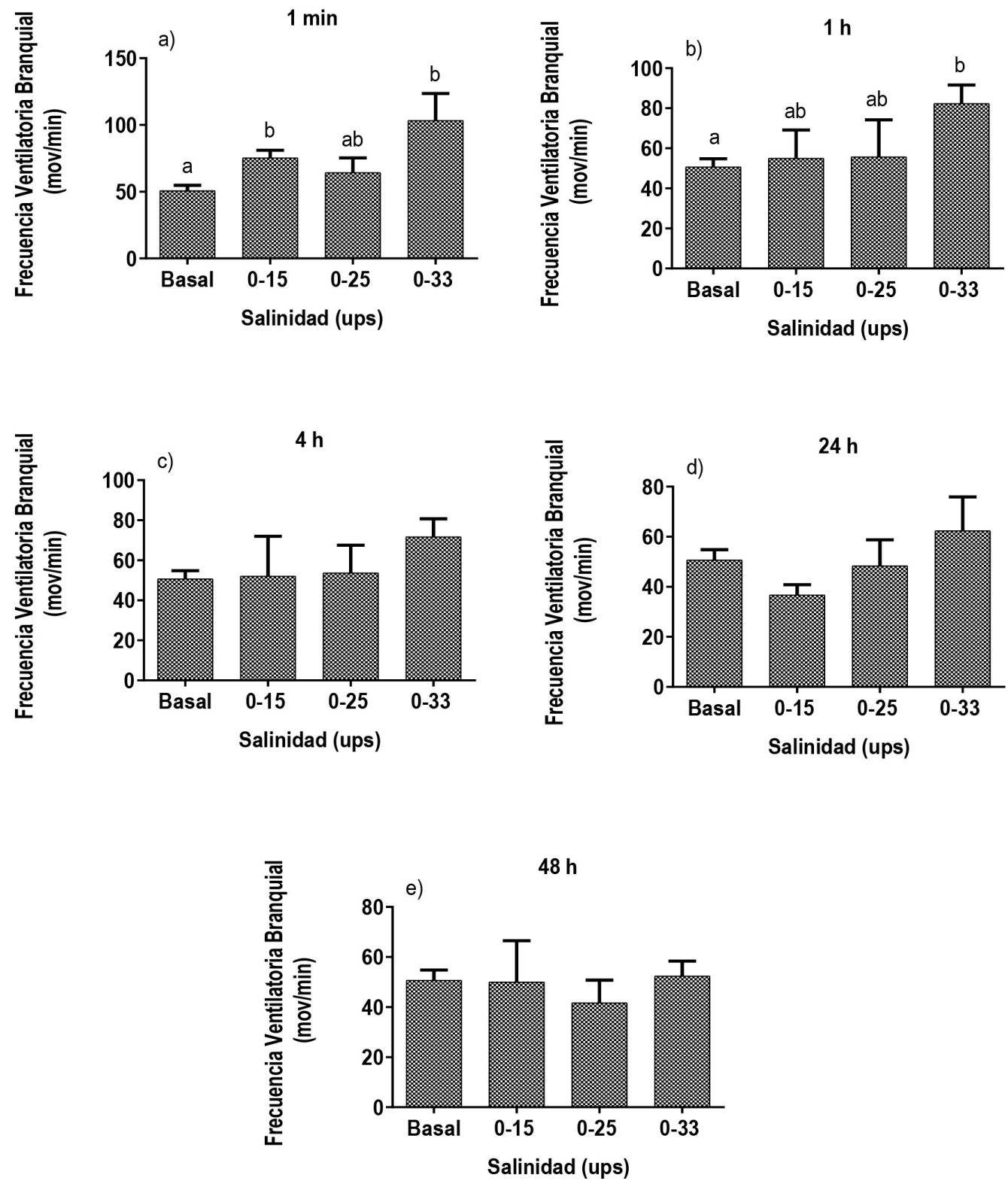

Figura 1. Frecuencia Ventiladora Branquial (FVB) de juveniles de $D$. latifrons transferidos de agua dulce hacia agua salada (0-15, 0-25, 0-33 ups), a), b), c), d) y e) FVB de los juveniles $D$. latifrons expuestos a los tratamientos con respecto al tiempo. Tratamientos con letras diferentes demuestran diferencias significativas $(p<0.05)$.

se encontraron diferencias significativas a las 4,24 y $48 \mathrm{~h}$, en el tratamiento 33-0.3 ups con respecto a la FVB basal con valor de $67.0 \pm 5.6$ a las $4 \mathrm{~h}$ y de $58.3 \pm 7.1$ ventilaciones por minuto a las 24 y $48 \mathrm{~h}$ de haber sido expuestos al cambio (Figura 2c, d y e).
Durante el proceso de aclimatación a distintas salinidades como en todos los casos de cambio salino se presentó un $100 \%$ de sobrevivencia. Lo que sugiere una alta capacidad de $D$. latifrons adaptarse a concentraciones salinas variables incluso soportar 

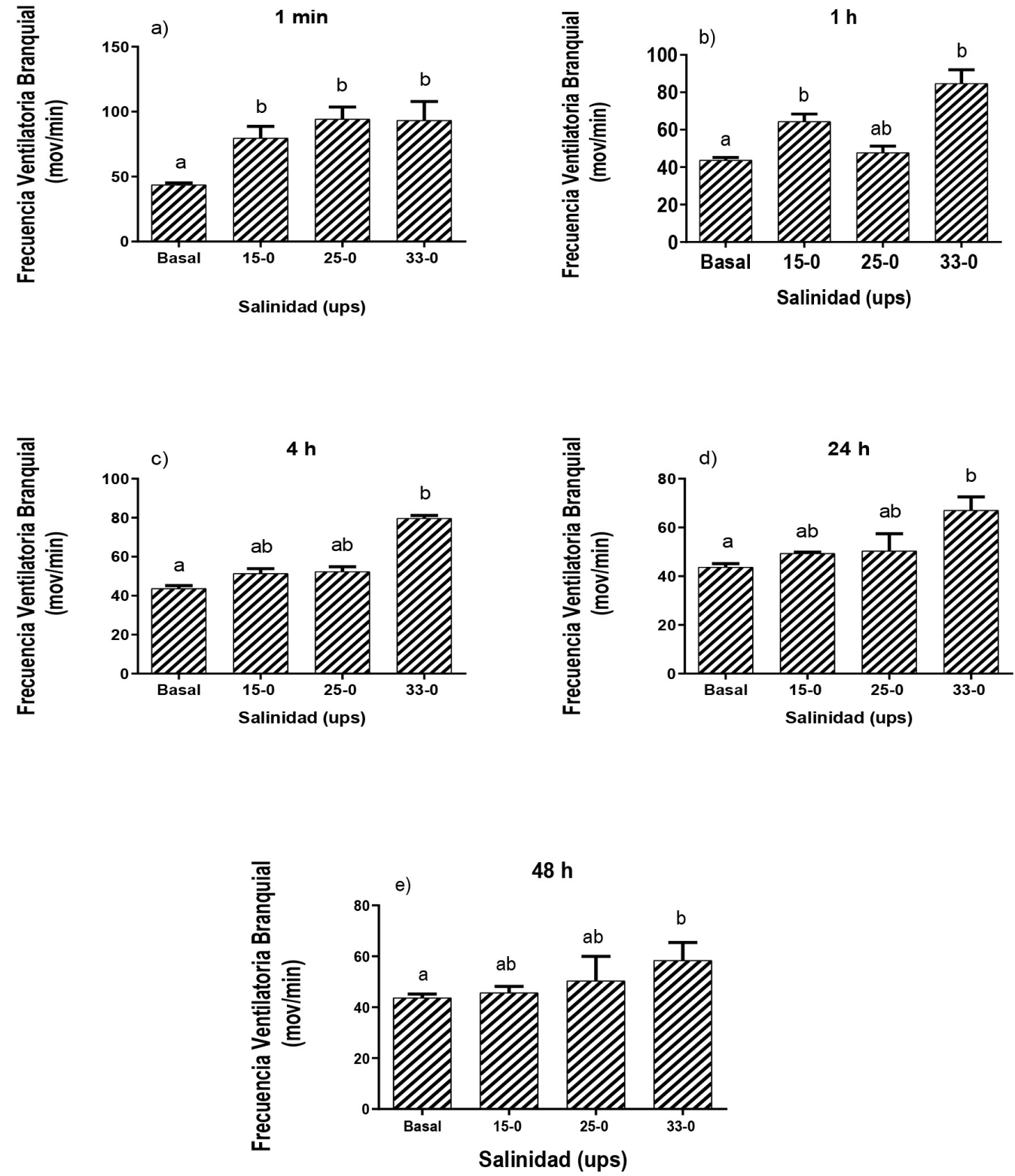

Figura 2. Frecuencia Ventilatoria Branquial (FVB) de juveniles de $D$. latifrons transferidos de agua salada hacia agua dulce (15-0, 25-0, 33-0 ups), a), b), c), d) y e) FVB de los juveniles $D$. latifrons expuestos a los tratamientos con respecto al tiempo. Tratamientos con letras diferentes demuestran diferencias significativas $(p<0.05)$.

cambios bruscos. Al respecto, se sabe que algunos peces eurihalinos tienen la capacidad fisiológica de adaptarse más rápido a los cambios en la salinidad del agua, compensando la situación acelerando el proceso de equilibrio osmótico (Bœuf y Payan 2001). Pero en el caso de que el proceso de adaptación no se lleve a cabo de manera rápida, el organismos entra en una fase de resistencia, en la que libera corticoides adrenales que pueden derivar en situaciones letales provocando la muerte de los organismos (Marrero-Hernández 2008). Al respecto, Nordlie y Haney (1993) evaluaron las tolerancias de 
salinidad y las concentraciones osmóticas de plasma para $D$. maculatus especie homóloga de $D$. latifrons en las costas del Atlántico americano, encontrando un límite de tolerancia entre 75 y 80 ups, con sobrevivencia del $47 \%$ de los peces sometidos a 80 ups. Sobre la tolerancia a la salinidad se sabe que la capacidad de un pez para aclimatarse a diferentes niveles de salinidad ambiental depende de su capacidad para regular la absorción y la excreción de iones, y mantener su equilibrio hidromineral (Zhao et al. 2011, Blancas-Arroyo et al. 2014). Los juveniles de $D$. latifrons fueron capaces de sobrevivir durante 30 días, tanto bajo condiciones de agua dulce (0.3 ups) como marinas (33 ups), sin aparente estrés; incluso bajo condiciones hiperosmóticas, cuando los peces retornaron a la FVB basal desde la primera hora, lo que confirma su carácter eurihalino.
Los resultados del presente estudio coinciden con los resultados de Nordlie y Haney (1993), con relación a las concentraciones secuenciales a las que fueron expuestos y la capacidad de regresar a la FVB basal al ser transferidos de agua salada ha agua dulce y viceversa con un $100 \%$ de sobrevivencia. Por lo que $D$. latifrons en un pez que muestra alta capacidad fisiológica de adaptación a ambientes con cambios salinos, por lo que se confirma su carácter eurihalino y su importancia ecológica al poder ocupar diversos nichos tróficos. Además, tales características lo hacen un buen candidato para ser cultivado en agua dulce y agua salobre. Por lo que se requiere ampliar los estudios sobre el efecto de la salinidad en el crecimiento, costo energético y fisiológico de $D$. latifrons, para establecer los márgenes osmóticos óptimos en los que puede desarrollarse de manera adecuada y sin estrés.

\section{LITERATURA CITADA}

Barreto RE, Luchiari AC, Marcondes AL (2003) Ventilatory frequency indicates visual recognition of an allopatric predator in naïve Nile tilapia. Behavioural Processes 60: 235-239.

Barreto RE, Volpato GL (2004) Caution for using ventilatory frequency as an indicator of stress in fish. Behavioural Processes 66: 43-51.

Becerra GE (1997) Respuestas osmoadaptativas del Guapote juvenil (Dormitator latifrons, Richardson, 1837) durante tranferencia directa al agua de mar. Tecnociencia 1: 50-56.

Blancas-Arroyo GA, Frías-Sevilla R, De La Rosa-Pimentel E, Suárez-Navarro V, Castro-Gómez JR, MagañaMorales J (2014) Efecto de la salinidad en la sobrevivencia de peces silvestres del género Chirostoma durante el transporte y mantenimiento en laboratorio. Hidrobiologica 24: 223-230.

Bœuf G, Payan P (2001) How should salinity influence fish growth? In: Grosell M (ed.) Comparative biochemistry and physiology - C Toxicology and pharmacology. Elsevier. Miami, Florida, USA. pp: 411-423.

Castro-Rivera R, Aguilar-Benítez G, Hernández-Girón JP (2005) Conversión alimenticia en engordas puras y mixtas de Popoyote (Dormitator latifrons Richardson) en estanques de cemento. Revista AquaTIC 23: 4552.

Chang BD (1984) Tolerances to salinity and air exposure of Dormitator latifrons (Pisces: Eleotridae). Revista Biología Tropical 32: 155-157.

Christensen EAF, Svendsen MBS, Steffensen JF (2017) Plasma osmolality and oxygen consumption of perch Perca fluviatilis in response to different salinities and temperatures. Journal Fish Biology 90: 819-833.

Corredor AS, Landines MA (2009) Efecto del ácido áscorbico sobre la respuesta de los peces ante condiciones de estrés. Revista de la Facultad de Medicina Veterinaria y de Zootecnia 56: 53-66.

Kültz D (2015) Physiological mechanisms used by fish to cope with salinity stress. Journal of Experimental Biology 218: 1907-1914. 
López-Huerta J, Vega-Villasante F, Viana M, Carrillo-Farnes O, Badillo-Zapata D (2018) First report of nutritional quality of the native fish Dormitator latifrons (Richardson, 1844) (Perciformes: Eleotridae). Latin American Journal of Aquatic Research 46: 849-854.

Marrero-Hernández MJ (2008) Estrés osmóticos en Chelon labrosus (Risso, 1827). Anales Universitarios de Etología 2: 56-61.

Nordlie FG, Haney DC (1998) Adaptations in salt marsh teleosts to life in waters of varying salinity. Italian Journal of Zoology 65: 405-409.

Nordlie FG, Haney DC (1993) Euryhaline adaptations in the fat sleeper, Dormitator maculatus. Journal of Fish Biology 43: 433-439.

Serrano SJ, Grosell M (2011) Osmoregulatory capabilities of the gray snapper, lutjanus griseus: Salinity challenges and field observations. Marine and Freshwater Behaviour and Physiology 44: 185-196.

Todd ES (1975) Vertical movements and development of the prolarvae of the eleotrid fish, Dormitator latifrons. Copeia 3: 564-568.

Vicuña O (2010) Dormitator latifrons (Chame). En: Flores-Nava A, Brown A (Ed.) Peces nativos de agua dulce de América del Sur de interés para la acuicultura: Una síntesis del estado de desarrollo tecnológico de su cultivo. FAO. Serie Acuicultura en Latinoamérica Número 1. Roma, Italia. pp: 63-70.

Yáñez-Arancibia A, Díaz-González G (1977) Trophic-dynamic ecology of Dormitator latifrons (Richardson) in nine coastal lagoons on the Pacific coast of Mexico. (Pisces: Electridae). Instituto de Ciencia del Mar y Limnología 19: 140.

Zhao F, Qu L, Zhuang P, Zhang L, Liu J, Zhang T (2011) Salinity tolerance as well as osmotic and ionic regulation in juvenile Chinese sturgeon (Acipenser sinensis Gray, 1835) exposed to different salinities. Journal of Applied Ichthyology 27: 231-234. 
